\title{
A note on solution lower semicontinuity for parametric generalized vector equilibrium problems
}

\author{
Zai Yun Peng ${ }^{1 *}$, Zhi Lin', Kai Zhi Yu² and Da Cheng Wang ${ }^{1}$
}

\section{"Correspondence:}

pengzaiyun@126.com

${ }^{1}$ College of Science, Chongqing

JiaoTong University, Chongqing,

400074, P.R. China

Full list of author information is

available at the end of the article

\section{基 Springer}

\begin{abstract}
By using a density technique, sufficient conditions for lower semicontinuity of strong solutions to a parametric generalized vector equilibrium problem are established, where the monotonicity is not necessary. The obtained results are different from the corresponding ones in the literature (Gong and Yao in J. Optim. Theory Appl. 138:197-205, 2008; Gong in J. Optim. Theory Appl. 139:35-46, 2008; Chen and Li in PaC. J. Optim. 6:141-151, 2010; Li and Fang in J. Optim. Theory Appl. 147:507-515, 2010; Gong and Yao in J. Optim. Theory Appl. 138:189-196, 2008). Some examples are given to illustrate the results.
\end{abstract}

MSC: 49K40; 90C29; 90C31

Keywords: stability; strong solution; lower semicontinuity; parametric vector equilibrium problem; density

\section{Introduction}

It is well known that the vector equilibrium problem (VEP, in short) is a very general mathematical model, which embraces the formats of several disciplines, as those for Nash equilibria, those from Game Theory, those from (Vector) Optimization and (Vector) Variational Inequalities, and so on (see [1-4]).

The stability analysis of solution maps for parametric vector equilibrium problems (PVEP, in short) is an important topic in optimization theory and applications. There are some papers discussing the upper and/or lower semicontinuity of solution maps. Cheng and Zhu [5] obtained a result on the lower semicontinuity of the solution set map to a PVEP in finite-dimensional spaces by using a scalarization method. Huang et al. [6] used local existence results of the models considered and additional assumptions to establish the lower semicontinuity of solution mappings for parametric implicit vector equilibrium problems. Recently, by virtue of a density result and scalarization technique, Gong and Yao [7] have first discussed the lower semicontinuity of efficient solutions to parametric vector equilibrium problems, which are called generalized systems in their paper. By using the idea of Cheng and Zhu [5], Gong [8] has discussed the continuity of the solution maps to a weak PVEP in Hausdorff topological vector spaces. Kimura and Yao [9] discussed the semicontinuity of solution maps for parametric vector quasi-equilibrium problems by virtue of the closedness or openness assumptions for some certain sets. $\mathrm{Xu}$ and $\mathrm{Li}$ [10] proved the lower semicontinuity for PVEP by using a new proof method, which is different 
from the one used in Gong and Yao [7]. Chen and Li [11] studied the continuity of solution sets for parametric generalized systems without the uniform compactness assumption, which improves the corresponding results in [8]. We observe that the semicontinuity of solution maps for the PVEP has been discussed under the assumption of $C$-strict (strong) monotonicity, which implies that the $f$-solution set of the PVEP is a singleton for a linear continuous functional $f$ (see $[5,7,8,10,11]$ ). However, it is well known that the $f$-solution set of (weak) PVEPs should be general, but not a singleton. Moreover, to the best of our knowledge, there are few results of semicontinuity have been established for strong solution maps of PVEP in the literature. So, in this paper, by using a density skill, we aim at studying the lower semicontinuity of the strong solution map for a class of parametric generalized vector equilibrium problems (PGVEPs), when the $f$-solution set is a general set by removing the assumption of $C$-strict monotonicity.

The rest of the paper is organized as follows. In Section 2, we introduce a class of parametric generalized vector equilibrium problem, and recall some concepts and their properties. In Section 3, by the density and scalarization technique, we discuss the lower semicontinuity of strong solution mappings to the PGVEP, and compare our main results with the corresponding ones in the recent literature $[7,8,10,11]$. We also give some examples to illustrate our results.

\section{Preliminaries}

Throughout this paper, unless specified otherwise, let $X, Y$ be normed spaces and $Z$ be Banach space. Let $Y^{*}$ be the topological dual space of $Y$, and $C$ be a closed convex pointed cone in $Y$ with nonempty topological interior int $C$.

Let

$$
C^{*}:=\left\{f \in Y^{*}: f(y) \geq 0, \forall y \in C\right\}
$$

be the dual cone of $C$. Denote the quasi-interior of $C^{*}$ by $C^{\sharp}$, i.e.,

$$
C^{\sharp}:=\left\{f \in Y^{*}: f(y)>0, \forall y \in C \backslash\{0\}\right\} .
$$

Let $A$ be a nonempty subset of $X$ and $F: A \times A \rightarrow Y$ be a vector-valued mapping. We consider the following generalized vector equilibrium problem:

Find $x \in A$ such that $F(x, y) \notin-K, \quad \forall y \in A$,

where $K \cup\{0\}$ is a convex cone in $Y$.

When the subset $A$ and the mapping $F$ are perturbed by a parameter $\mu \in \Lambda$, in which $\Lambda$ is a nonempty subset of $Z$, we consider the following parametric generalized vector equilibrium problem (PGVEP):

Find $x \in A(\mu)$ such that $F(x, y, \mu) \notin-K, \quad \forall y \in A(\mu)$,

where $A: \Lambda \rightarrow 2^{X} \backslash\{\emptyset\}$ is a set-valued mapping, $F: B \times B \times \Lambda \subset X \times X \times Z \rightarrow Y$ is a vector-valued mapping with $A(\Lambda)=\bigcup_{\mu \in \Lambda} A(\mu) \subset B$. 
Definition 2.1 [12] A vector $x \in A(\mu)$ is called a weak solution to the (PGVEP), iff

$$
F(x, y, \mu) \notin-\operatorname{int} C, \quad \forall y \in A(\mu) .
$$

The set of the weak solutions to the (PGVEP) is denoted by $V_{w}(\mu)$.

Definition 2.2 A vector $x \in A(\mu)$ is called a strong solution to the (PGVEP), iff

$$
F(x, y, \mu) \notin-C \backslash\{0\}, \quad \forall y \in A(\mu) .
$$

The set of the strong solutions to the (PGVEP) is denoted by $V_{s}(\mu)$.

Definition 2.3 [7] Let $f \in C^{*} \backslash\{0\}$. A vector $x \in A(\mu)$ is called an $f$-solution to the (PGVEP), iff

$$
f(F(x, y, \mu)) \geq 0, \quad \forall y \in A(\mu) .
$$

The set of the $f$-solution to the (PGVEP) is denoted by $V_{f}(\mu)$.

Definition 2.4 Let $F: X \times X \times \Lambda \rightarrow Y$ be a vector-valued mapping.

(i) $F(\cdot, \cdot, \cdot)$ is called $C$-monotone on $A(\mu) \times A(\mu) \times \Lambda$, iff for any given $\mu \in \Lambda$, for each $x, y \in A(\mu), F(x, y, \mu)+F(y, x, \mu) \in-C$.

(ii) $F(\cdot,,, \cdot)$ is called $C$-strictly monotone (i.e., $C$-strongly monotone in [13]) on $A(\mu) \times A(\mu) \times \Lambda$, iff $F$ is a $C$-monotone on $A(\mu) \times A(\mu) \times \Lambda$, and for any given $\mu \in \Lambda$, for each $x, y \in A(\mu)$ with $x \neq y, F(x, y, \mu)+F(y, x, \mu) \in-\operatorname{int} C$.

(iii) $F(x, \cdot, \mu)$ is called $C$-convex if, for each $x_{1}, x_{2} \in A(\mu)$ and $t \in[0,1]$, $t F\left(x, x_{1}, \mu\right)+(1-t) F\left(x, x_{2}, \mu\right) \in F\left(x, t x_{1}+(1-t) x_{2}, \mu\right)+C$.

(iv) $F(x, \cdot, \mu)$ is called $C$-like-convex on $A(\mu)$, iff for any $x_{1}, x_{2} \in A(\mu)$ and any $t \in[0,1]$, there exists $x_{3} \in A(\mu)$ such that $t F\left(x, x_{1}, \mu\right)+(1-t) F\left(x, x_{2}, \mu\right) \in F\left(x, x_{3}, \mu\right)+C$.

(v) A set $D \subset Y$ is called a $C$-convex set, iff $D+C$ is a convex set in $Y$.

Throughout this paper, we always assume $V_{w}(\mu) \neq \emptyset$ and $V_{s}(\mu) \neq \emptyset$ for all $\mu \in \Lambda$. This paper aims at investigating the semicontinuity of the strong solution mappings to (PGVEP).

Lemma 2 .1 [14] Let $F(x, A(\mu), \mu)$ be a $C$-convex set for each $\mu \in \Lambda$ and $x \in A(\mu)$. If int $C \neq \emptyset$, then $V_{w}(\mu)=\bigcup_{f \in C^{*} \backslash\{0\}} V_{f}(\mu)$.

The notation $B(\bar{\lambda}, \delta)$ denotes the open ball with center $\bar{\lambda} \in \Lambda$ and radius $\delta>0$. Let $F$ : $\Lambda \rightarrow 2^{X}$ be a set-valued mapping, and let there be given $\bar{\lambda} \in \Lambda$.

\section{Definition 2.5 [15]}

(i) $F$ is called lower semicontinuous (l.s.c., for short) at $\bar{\lambda}$, iff for any open set $V$ satisfying $V \cap F(\bar{\lambda}) \neq \emptyset$, there exists $\delta>0$, such that for every $\lambda \in B(\bar{\lambda}, \delta)$, $V \cap F(\lambda) \neq \emptyset$.

(ii) $F$ is called upper semicontinuous (u.s.c., for short) at $\bar{\lambda}$, iff for any open set $V$ satisfying $F(\bar{\lambda}) \subset V$, there exists $\delta>0$, such that for every $\lambda \in B(\bar{\lambda}, \delta), F(\lambda) \subset V$. 
We say $F$ is l.s.c. (resp. u.s.c.) on $\Lambda$, iff it is l.s.c. (resp. u.s.c.) at each $\lambda \in \Lambda$. $F$ is said to be continuous on $\Lambda$, iff it is both l.s.c. and u.s.c. on $\Lambda$.

\section{Proposition 2.1 [15]}

(i) $F$ is l.s.c. at $\bar{\lambda}$ if and only iffor any sequence $\left\{\lambda_{n}\right\} \subset \Lambda$ with $\lambda_{n} \rightarrow \bar{\lambda}$ and any $\bar{x} \in F(\bar{\lambda})$, there exists $x_{n} \in F\left(\lambda_{n}\right)$, such that $x_{n} \rightarrow \bar{x}$.

(ii) If $F$ has compact values (i.e., $F(\lambda)$ is a compact set for each $\lambda \in \Lambda$ ), then $F$ is u.s.c. at $\bar{\lambda}$ if and only iffor any sequences $\left\{\lambda_{n}\right\} \subset \Lambda$ with $\lambda_{n} \rightarrow \bar{\lambda}$ and $\left\{x_{n}\right\}$ with $x_{n} \in F\left(\lambda_{n}\right)$, there exist $\bar{x} \in F(\bar{\lambda})$ and a subsequence $\left\{x_{n_{k}}\right\}$ of $\left\{x_{n}\right\}$, such that $x_{n_{k}} \rightarrow \bar{x}$.

The following lemma plays an important role in the proof of the lower semicontinuity of the solution map $V_{s}(\mu)$.

Lemma 2.2 [16, Theorem 2, p.114] The union $\Gamma=\bigcup_{i \in I} \Gamma_{i}$ of a family of l.s.c. set-valued mappings $\Gamma_{i}$ from a topological space $X$ into a topological space $Y$ is also an l.s.c.set-valued mapping from $X$ into $Y$, where $I$ is an index set.

Let $Q: X \rightarrow 2^{Y}$ be a set-valued mapping between two topological spaces. The lower limit of $Q$ is defined as

$$
\operatorname{Liminf}_{x \rightarrow x_{0}} Q(x)=\left\{y \in Y: \forall x_{\alpha} \rightarrow x_{0}, \exists y_{\alpha} \in Q\left(x_{\alpha}\right), y_{\alpha} \rightarrow y\right\} .
$$

\section{Proposition 2.2 [17]}

(i) Liminf $_{x \rightarrow x_{0}} Q(x)$ is a closed set.

(ii) $Q$ is l.s.c. at $x_{0} \in \operatorname{dom} Q:=\{x \mid Q(x) \neq \emptyset\}$ if and only if $Q\left(x_{0}\right) \subset \operatorname{Liminf}_{x \rightarrow x_{0}} Q(x)$.

\section{Lower semicontinuity of strong solution sets to (PGVEP)}

In this section, we discuss the lower semicontinuity of the strong solutions for (PVEP). Firstly, we obtain two important lemmas relevant to the (PVEP) as follows.

Lemma 3.1 Let $f \in C^{*} \backslash\{0\}$. Suppose the following conditions are satisfied:

(i) $A(\cdot)$ is continuous with compact convex values on $\Lambda$.

(ii) For each $\mu \in \Lambda, F(\cdot, \cdot, \cdot)$ is continuous on $B \times B \times \mu$.

(iii) For each $\mu \in \Lambda, x \in A(\mu) \backslash V_{f}(\mu)$, there exist $y \in V_{f}(\mu)$ and $r>0$, such that

$$
F(x, y, \mu)+F(y, x, \mu)+B\left(0, d^{r}(x, y)\right) \subset-C .
$$

Then $V_{f}(\cdot)$ is l.s.c. on $\Lambda$.

Proof Suppose to the contrary that there exists $\mu_{0} \in \Lambda$, such that $V_{f}(\cdot)$ is not l.s.c. at $\mu_{0}$. Then there exist a sequence $\left\{\mu_{n}\right\}$ with $\mu_{n} \rightarrow \mu_{0}$ and $x_{0} \in V_{f}\left(\mu_{0}\right)$, such that for any $x_{n} \in$ $V_{f}\left(\mu_{n}\right), x_{n} \nrightarrow x_{0}$.

Since $A(\cdot)$ is l.s.c. at $\mu_{0}$, there exists $\bar{x}_{n} \in A\left(\mu_{n}\right)$, such that $\bar{x}_{n} \rightarrow x_{0}$. Obviously, $\bar{x}_{n} \in$ $A\left(\mu_{n}\right) \backslash V_{f}\left(\mu_{n}\right)$. By (iii), there exists $y_{n} \in V_{f}\left(\mu_{n}\right)$ such that

$$
F\left(\bar{x}_{n}, y_{n}, \mu_{n}\right)+F\left(y_{n}, \bar{x}_{n}, \mu_{n}\right)+B\left(0, d^{r}\left(\bar{x}_{n}, y_{n}\right)\right) \subset-C .
$$


For $y_{n} \in A\left(\mu_{n}\right)$, because $A(\cdot)$ is u.s.c. at $\mu_{0}$ with compact values, there exist $y_{0} \in A\left(\mu_{0}\right)$ and a subsequence $\left\{y_{n_{k}}\right\}$ of $\left\{y_{n}\right\}$, such that $y_{n_{k}} \rightarrow y_{0}$. In particular, for (3.1), we have

$$
F\left(\bar{x}_{n_{k}}, y_{n_{k}}, \mu_{n_{k}}\right)+F\left(y_{n_{k}}, \bar{x}_{n_{k}}, \mu_{n_{k}}\right)+B\left(0, d^{r}\left(\bar{x}_{n_{k}}, y_{n_{k}}\right)\right) \subset-C .
$$

Taking the limit as $n_{k} \rightarrow+\infty$, we have

$$
F\left(x_{0}, y_{0}, \mu_{0}\right)+F\left(y_{0}, x_{0}, \mu_{0}\right)+B\left(0, d^{r}\left(x_{0}, y_{0}\right)\right) \subset-C .
$$

Noting that $x_{0} \in V_{f}\left(\mu_{0}\right)$ and $y_{0} \in A\left(\mu_{0}\right)$, we have

$$
f\left(F\left(x_{0}, y_{0}, \mu_{0}\right)\right) \geq 0 \text {. }
$$

Moreover, since $y_{n_{k}} \in V_{f}\left(\mu_{n_{k}}\right)$ and $\bar{x}_{n_{k}} \in A\left(\mu_{n_{k}}\right)$, it follows from the continuity of $f$ and $F$ that

$$
f\left(F\left(y_{0}, x_{0}, \mu_{0}\right)\right) \geq 0
$$

By (3.4), (3.5), and the linearity of $f$, we have

$$
f\left(F\left(x_{0}, y_{0}, \mu_{0}\right)+F\left(y_{0}, x_{0}, \mu_{0}\right)\right) \geq 0 .
$$

Assume that $x_{0} \neq y_{0}$; by (3.3), we obtain $F\left(x_{0}, y_{0}, \mu_{0}\right)+F\left(y_{0}, x_{0}, \mu_{0}\right) \subset-\operatorname{int} C$. Thus, we have

$$
f\left(F\left(x_{0}, y_{0}, \mu_{0}\right)+F\left(y_{0}, x_{0}, \mu_{0}\right)\right)<0,
$$

which contradicts (3.6). Therefore $x_{0}=y_{0}$, which leads to a contradiction. Hence, for each $f \in C^{*} \backslash\{0\}, S_{f}(\cdot)$ is l.s.c. on $\Lambda$.

Lemma 3.2 Let $f \in C^{*} \backslash\{0\}$. Suppose the following conditions are satisfied:

(i) $A$ is nonempty compact set.

(ii) $F(\cdot, \cdot)$ is continuous on $B \times B$.

(iii) For each $x \in A \backslash V_{f}$ there exist $y \in V_{f}$ and $r>0$, such that

$$
F(x, y)+F(y, x)+B\left(0, d^{r}(x, y)\right) \subset-C
$$

Let us define the set-valued mapping $H: C^{*} \backslash\{0\} \rightarrow 2^{A}$ by

$$
H(f)=V_{f}, \quad f \in C^{*} \backslash\{0\}
$$

then we see that $H(\cdot)$ is l.s.c. on $C^{*} \backslash\{0\}$.

Proof By using Lemma 3.1 and following a similar way to the proof to Lemma 3.1 of [18], we can obtain the conclusion.

Now, we discuss the lower semicontinuity of strong solution mappings to (PGVEP). 
Theorem 3.1 Let $f \in C^{*} \backslash\{0\}$. Suppose the following conditions are satisfied:

(i) $A(\cdot)$ is continuous with compact convex values on $\Lambda$.

(ii) For each $\mu \in \Lambda$ and $x \in A(\mu), F(x, \cdot, \mu)$ is $C$-like-convex on $A(\mu)$.

(iii) For each $\mu \in \Lambda, F(\cdot, \cdot, \cdot)$ is continuous on $B \times B \times \mu$.

(iv) For each $\mu \in \Lambda, x \in A(\mu) \backslash V_{f}(\mu)$, there exist $y \in V_{f}(\mu)$ and $r>0$, such that

$$
F(x, y, \mu)+F(y, x, \mu)+B\left(0, d^{r}(x, y)\right) \subset-C .
$$

(v) $F(A(\mu), A(\mu), \mu)$ are bounded subsets of $Y$ for each $\mu \in \Lambda$.

(vi) $C^{\sharp} \neq \emptyset$ and int $C \neq \emptyset$.

Then $V_{s}(\cdot)$ is lower semicontinuous on $\Lambda$.

Proof We prove the result in the following three steps.

Step 1. We first show that

$$
\bigcup_{f \in C^{\sharp}} V_{f}(\mu) \subset V_{s}(\mu) \subset c l\left(\bigcup_{f \in C^{\sharp}} V_{f}(\mu)\right) .
$$

Since $V_{f}(\mu) \neq \emptyset$, for each $f \in C^{*} \backslash\{0\}$. Then, by definition, we have

$$
\bigcup_{f \in C^{\sharp}} V_{f}(\mu) \subset V_{s}(\mu) \subset V_{w}(\mu) .
$$

Since for any $x \in A(\mu), F(x, \cdot, \mu)$ is $C$-like-convex, then $F(x, A(\mu), \mu)+C$ is a convex set. From Lemma 2.1, we have

$$
V_{w}(\mu)=\bigcup_{f \in C^{*} \backslash\{0\}} V_{f}(\mu)
$$

By (3.7) and (3.8), we get

$$
\bigcup_{f \in C^{\sharp}} V_{f}(\mu) \subset V_{s}(\mu) \subset \bigcup_{f \in C^{*} \backslash\{0\}} V_{f}(\mu) .
$$

To show that

$$
\bigcup_{f \in C^{*} \backslash\{0\}} V_{f}(\mu) \subset c l\left(\bigcup_{f \in C^{\sharp}} V_{f}(\mu)\right),
$$

we first prove

$$
\bigcup_{f \in C^{*} \backslash\{0\}} V_{f} \subset c l\left(\bigcup_{f \in C^{\sharp}} V_{f}\right) .
$$

Let us define the set-valued mapping $H: C^{*} \backslash\{0\} \rightarrow 2^{A}$ by

$$
H(f)=V_{f}, \quad f \in C^{*} \backslash\{0\} .
$$

By Lemma 3.2, we know that $H(\cdot)$ is lower semicontinuous on $C^{*} \backslash\{0\}$. 
Let $x_{0} \in \bigcup_{f \in C^{*} \backslash\{0\}} V_{f}$. Then there exists $f_{0} \in C^{*} \backslash\{0\}$ such that

$$
x_{0} \in V_{f_{0}}=H\left(f_{0}\right) \text {. }
$$

Since $C^{\sharp} \neq \emptyset$, let $g \in C^{\sharp}$ and set

$$
f_{n}=f_{0}+(1 / n) g
$$

Then $f_{n} \in C^{\sharp}$. We show that $\left\{f_{n}\right\}$ converges to $f_{0}$ with respect to the topology $\beta\left(Y^{*}, Y\right)$.

For any neighborhood $U$ of 0 with respect to $\beta\left(Y^{*}, Y\right)$, there exist bounded subsets $B_{i} \subset$ $Y(i=1,2, \ldots, m)$ and $\epsilon>0$ such that

$$
\bigcap_{i=1}^{m}\left\{f \in Y^{*}: \sup _{y \in B_{i}}|f(y)|<\epsilon\right\} \subset U
$$

Since $B_{i}$ is bounded and $g \in Y^{*},\left|g\left(B_{i}\right)\right|$ is bounded for $i=1, \ldots, m$. Thus, there exists $N$ such that

$$
\sup _{y \in B_{i}}|(1 / n) g(y)|<\epsilon, \quad i=1, \ldots, m, n \geq N .
$$

Hence $(1 / n) g \in U$, that is, $f_{n}-f_{0} \in U$. This means that $\left\{f_{n}\right\}$ converges to $f_{0}$ with respect to $\beta\left(Y^{*}, Y\right)$.

Since $H(f)$ is l.s.c. at $f_{0}$, for sequence $\left\{f_{n}\right\} \subset C^{*} \backslash\{0\}, f_{n} \rightarrow f_{0}$ and $x_{0} \in H\left(f_{0}\right)$, there exists $x_{n} \in H\left(f_{n}\right)=V_{f_{n}} \subset \bigcup_{f \in C^{\sharp}} V_{f}$, such that $x_{n} \rightarrow x_{0}$. This means that

$$
x_{0} \in c l\left(\bigcup_{f \in C^{\sharp}} V_{f}\right) .
$$

By the arbitrariness of $x_{0} \in \bigcup_{f \in C^{*} \backslash\{0\}} V_{f}$, we have

$$
\bigcup_{f \in C^{*} \backslash\{0\}} V_{f} \subset c l\left(\bigcup_{f \in C^{\sharp}} V_{f}\right) .
$$

Then we can obtain the result that for each $\mu \in \Lambda$ (3.11) is valid, and the validity of (3.7) follows readily from (3.10) and (3.11).

Step 2. For each $\mu \in \Lambda$, let $\tilde{S}(\mu):=\bigcup_{f \in C^{\sharp}} V_{f}(\mu)$. By a similar argument to the proof of Lemma 3.1, we find for each $f \in C^{\sharp}$, that $V_{f}(\cdot)$ is l.s.c. on $\Lambda$. It follows from Lemma 2.2 that $\tilde{S}(\cdot)$ is l.s.c. on $\Lambda$.

Step 3. Now we show that $V_{s}(\cdot)$ is lower semicontinuous on $\Lambda$. From Step 1, we have

$$
\tilde{S}(\mu) \subset V_{s}(\mu) \subset \operatorname{cl}(\tilde{S}(\mu)), \quad \forall \mu \in \Lambda .
$$

Let $\mu_{0} \in \Lambda$ be any fixed point. Because of the lower semicontinuity of $\tilde{S}(\cdot)$ at $\mu_{0}$ and the closedness of the lower limit of $\tilde{S}$ (see Proposition 2.2), together with the above inclusion relation, we have

$$
V_{s}\left(\mu_{0}\right) \subset \operatorname{cl}\left(\tilde{S}\left(\mu_{0}\right)\right) \subset \liminf _{\mu_{\alpha} \rightarrow \mu_{0}} \tilde{S}\left(\mu_{\alpha}\right) \subset \liminf _{\mu_{\alpha} \rightarrow \mu_{0}} V_{s}\left(\mu_{\alpha}\right) .
$$


Hence, $V_{s}(\cdot)$ is l.s.c. at $\mu_{0}$. By the arbitrariness of $\mu_{0}, V_{s}(\cdot)$ is l.s.c. on $\Lambda$. This completes the proof.

Remark 3.2 In Theorem 3.1, by using the density technique, we obtain a sufficient condition for the lower semicontinuity of strong solutions to (PGVEP). Our approach is different from the corresponding ones in [7] (see Theorem 2.1 of [7]). Furthermore, the condition of $C$-strong (strict) monotonicity is not required, and the $C$-convexity of $F$ is generalized to the $C$-like-convexity. Thus, Theorem 3.1 improves and extends the corresponding results in the literature $[5,7,8,10,11]$. The following example is given to illustrate the case.

Example 3.1 Let $X=R, Y=R^{2}, C=R_{+}^{2}, \Lambda=[1,2], A(\mu)=[-1,1]$, and $F(x, y, \mu)=\left(1-2 \mu^{2}+\right.$ $y, 2 \mu x)$. For any $f \in C^{*} \backslash\{0\}$, it follows from a direct computation that $V_{f}(\mu)=[0,1]$. It is clear that $A(\cdot)$ is a continuous set-valued mapping with nonempty compact convex values and for each $\mu \in \Lambda, F(\cdot, \cdot, \cdot)$ is continuous on $A(\mu) \times A(\mu) \times \mu$. Therefore, conditions (i)(ii) of Theorem 3.1 are satisfied. For any given $\mu \in \Lambda$ and for any given $x \in A(\mu)$, we have, for any $y_{1}, y_{2} \in A(\mu), t \in[0,1]$,

$$
\begin{aligned}
& F\left(x, t y_{1}+(1-t) y_{2}, \mu\right) \\
& \quad=\left(1-2 \mu^{2}+t y_{1}+(1-t) y_{2}, 2 \mu x\right) \\
& \quad=t\left(1-2 \mu^{2}+y_{1}, 2 \mu x\right)+(1-t)\left(1-2 \mu^{2}+y_{2}, 2 \mu x\right) \\
& \quad \in t F\left(x, y_{1}, \mu\right)+(1-t) F\left(x, y_{2}, \mu\right)-C .
\end{aligned}
$$

Thus, for any given $\mu \in \Lambda$ and for any given $x \in A(\mu), F(x, \cdot, \mu)$ is $R_{+}^{2}$-like-convex on $A(\mu)$, that is, condition (iii) of Theorem 3.1 is satisfied.

For any $x \in A(\mu) \backslash V_{f}(\mu)$, there exists $y=0 \in V_{f}(\mu)$, such that

$$
\begin{aligned}
& F(x, y, \mu)+F(y, x, \mu)+B\left(0, d^{r}(x, y)\right) \\
& \quad=\left(2-4 \mu^{2}+x, 2 \mu x\right)+B\left(0, d^{r}(x, 0)\right) \subset-C .
\end{aligned}
$$

Thus, the condition (iv) of Theorem 3.1 is satisfied. It is clear that $F(A(\mu), A(\mu), \mu)$ are bounded subsets of $Y$ for each $\mu \in \Lambda,\left(R_{+}^{2}\right)^{\sharp} \neq \emptyset$, and int $R_{+}^{2} \neq \emptyset$. Consequently, by Theorem 3.1, $V_{s}(\cdot)$ is lower semicontinuous on $\Lambda$.

However, the condition of $C$-strong monotonicity does not hold. Indeed, for any $x \in$ $A(\mu) \backslash V_{f}(\mu)=[-1,0)$, there exists $y=-x \in V_{f}(\mu)=[0,1]$, such that

$$
F(x, y, \mu)+F(y, x, \mu)=\left(-4 \mu^{2}+2,0\right) \notin-\operatorname{int} C .
$$

Obviously, $F(\cdot, \cdot, \mu)$ is not $C$-strongly monotone on $A(\mu) \times A(\mu)$. Then Theorem 2.1 in [7] and Theorem 3.2 in [11] are not applicable, and the corresponding results in references [8, Theorem 4.2], [10, Theorem 3.1] are also not applicable.

Finally, we give an example to illustrate that the assumption (iv) of Theorem 3.1 is essential. 
Example 3.2 Let $X=R, Y=R^{2}, C=R_{+}^{2}$. Let $\Lambda=[4,5]$ and

$$
A(\mu)=[\mu-4,1], \quad \mu \in \Lambda .
$$

It is clear that $A$ is a continuous set-valued mapping from $\Lambda$ to $X$ with nonempty compact convex values. Define the mapping $F: A(\mu) \times A(\mu) \times \Lambda \rightarrow R^{2}$ by

$$
F(x, y, \mu)=\left(\left(30-\mu^{2}\right) \frac{\mu}{5} x(x-y), 0\right), \quad \forall x, y \in A(\mu), \mu \in \Lambda .
$$

Obviously, we know that conditions (ii), (iii), (v), and (vi) are satisfied.

We have, for any $f \in C^{*} \backslash\{0\}, 0 \in V_{f}(\mu)$, and

$$
V_{s}(\mu)=V_{f}(\mu)=\{0,1\}, \quad \text { if } \mu=4 \quad \text { and } \quad V_{s}(\mu)=\{1\}, \quad \text { if } \mu \in(4,5] .
$$

In fact, there exists $x=\frac{1}{2} \in A(\mu) \backslash V_{f}(\mu)$, for $y=0 \in V_{f}(\mu)$, we have

$$
\begin{aligned}
& F(x, y, \mu)+F(y, x, \mu)+B\left(0, d^{r}(x, y)\right) \\
& \quad=\left(\frac{14}{5}, 0\right)+B\left(0, d^{r}\left(\frac{1}{2}, 0\right)\right) \not \subset-C .
\end{aligned}
$$

Using a similar method, there exists $x=\frac{1}{2} \in A(\mu) \backslash V_{f}(\mu)$, for $y=1 \in V_{f}(\mu)$, we have $F(x, y, \mu)+F(y, x, \mu)+B\left(0, d^{r}(x, y)\right) \not \subset-C$. Thus, condition (iv) of Theorem 3.1 is not satisfied.

Now, we show that $V_{s}(\mu)$ is not lower semicontinuous at $\mu_{0}=4$. There exists $0 \in V_{s}(4)$ and there exists a neighborhood $\left(-\frac{1}{2}, \frac{1}{2}\right)$ of 0 , for any neighborhood $U(4)$ of 4 , there exists $4<\tilde{\mu}<5$ such that $\tilde{\mu} \in U(4)$ and

$$
V_{s}(\tilde{\mu})=1 \notin\left(-\frac{1}{2}, \frac{1}{2}\right) .
$$

Thus,

$$
V_{s}(\tilde{\mu}) \cap\left(-\frac{1}{2}, \frac{1}{2}\right)=\emptyset .
$$

By virtue of Definition 2.5, we know that $V_{s}(\mu)$ is not lower semicontinuous at $\mu_{0}=4$.

\section{Competing interests}

The authors declare that they have no competing interests.

\section{Authors' contributions}

All authors contributed equally to the writing of this paper. All authors read and approved the final manuscript.

\section{Author details}

${ }^{1}$ College of Science, Chongqing JiaoTong University, Chongqing, 400074, P.R. China. ${ }^{2}$ Statistics School, Southwestern University of Finance and Economics, Chengdu, 611130, China.

\section{Acknowledgements}

This work was supported by the Natural Science Foundation of China (Nos. 11301571, 11271389, 71201126), the science foundation of Ministry of Education of China (No. 12XJC910001), the Natural Science Foundation Project of ChongQing (No. 2012jjA00016) and the Education Committee Research Foundation of Chongqing (KJ130428). 


\section{References}

1. Fan, K: Extensions of two fixed point theorems of F.E. Browder. Math. Z. 112, 234-240 (1969)

2. Fan, K: A minimax inequality and applications. In: Shihsha, O (ed.) Inequality, vol. III, pp. 103-113. Academic Press, New York (1972)

3. Giannessi, F: Vector Variational Inequalities and Vector Equilibria. Mathematical Theories. Kluwer, Dordrecht (2000)

4. Hou, SH, Gong, XH, Yang, XM: Existence and stability of solutions for generalized Ky Fan inequality problems with trifunctions. J. Optim. Theory Appl. 146, 387-398 (2010)

5. Cheng, YH, Zhu, DL: Global stability results for the weak vector variational inequality. J. Glob. Optim. 32, 543-550 (2005)

6. Huang, NJ, Li, J, Thompson, HB: Stability for parametric implicit vector equilibrium problems. Math. Comput. Model. 43, 1267-1274 (2006)

7. Gong, XH, Yao, JC: Lower semicontinuity of the set of efficient solutions for generalized systems. J. Optim. Theory Appl. 138, 197-205 (2008)

8. Gong, XH: Continuity of the solution set to parametric weak vector equilibrium problems. J. Optim. Theory Appl. 139, 35-46 (2008)

9. Kimura, K, Yao, JC: Sensitivity analysis of solution mappings of parametric vector quasi-equilibrium problems. J. Glob. Optim. 41, 187-202 (2008)

10. $\mathrm{Xu}, \mathrm{S}, \mathrm{Li}, \mathrm{SJ}$ : A new proof approach to lower semicontinuity for parametric vector equilibrium problems. Optim. Lett. 3, 453-459 (2009)

11. Chen, CR, Li, SJ: On the solution continuity of parametric generalized systems. Pac. J. Optim. 6, 141-151 (2010)

12. Gong, XH: Efficiency and Henig efficiency for vector equilibrium problems. J. Optim. Theory Appl. 108, 139-154 (2001)

13. Gong, XH, Yao, JC: Connectedness of the set of efficient solution for generalized systems. J. Optim. Theory Appl. 138, 189-196 (2008)

14. Gong, XH: Connectedness of the solution sets and scalarization for vector equilibrium problems. J. Optim. Theory Appl. 133, 151-161 (2007)

15. Aubin, JP, Ekeland, I: Applied Nonlinear Analysis. Wiley, New York (1984)

16. Aubin, JP, Frankowska, H: Set-Valued Analysis. Birkhäuser, Boston (1990)

17. Anh, LQ, Khanh, PQ: Semicontinuity of the solution sets to parametric quasivariational inclusions with applications to traffic networks I: upper semicontinuities. Set-Valued Anal. 16, 267-279 (2008)

18. Peng, $Z Y$, Yang, $X M$ : On the connectedness of efficient solutions for generalized Ky Fan inequality problems. J. Nonlinear Convex Anal. (accepted)

10.1186/1029-242X-2014-325

Cite this article as: Peng et al.: A note on solution lower semicontinuity for parametric generalized vector equilibrium problems. Journal of Inequalities and Applications 2014, 2014:325

\section{Submit your manuscript to a SpringerOpen ${ }^{0}$ journal and benefit from:}

- Convenient online submission

- Rigorous peer review

Immediate publication on acceptance

- Open access: articles freely available online

- High visibility within the field

- Retaining the copyright to your article 